\title{
Electroacupuncture improves cognitive ability following cerebral ischemia reperfusion injury via CaM-CaMKIV-CREB signaling in the rat hippocampus
}

\author{
YUN ZHANG ${ }^{1,2 *}$, RUHUI LIN ${ }^{1 *}$, JING TAO $^{1}$, YUNAN WU $^{3}$, BIN CHEN $^{4}$, \\ KUNQIANG $\mathrm{YU}^{4}$, JIXIANG CHEN ${ }^{4},{\mathrm{XIAOJIE} \mathrm{LI}^{3} \text { and LI-DIAN CHEN }}^{3}$ \\ ${ }^{1}$ College of Rehabilitation Medicine, Fujian University of Traditional Medicine, Fuzhou, Fujian 350122; \\ ${ }^{2}$ The Clinical Medicine Department, Fujian Health College, Fuzhou, Fujian 350101; \\ ${ }^{3}$ Fujian Key Laboratory of Exercise Rehabilitation, Fujian University of Traditional Chinese Medicine; \\ ${ }^{4}$ Traditional Chinese Medicine Rehabilitation Research Center, State Administration of Fujian \\ University of Traditional Chinese Medicine, Fuzhou, Fujian 350122, P.R. China
}

Received April 1, 2015; Accepted May 22, 2015

DOI: $10.3892 /$ etm.2016.3428

\begin{abstract}
The aim of the present study was to investigate the effect of electroacupuncture (EA) on cognitive deficits, and the underlying mechanism following cerebral ischemia-reperfusion $(\mathrm{I} / \mathrm{R})$ via the calmodulin $(\mathrm{CaM})$-calmodulin-dependent protein kinase type IV (CaMKIV)-cyclic adenosine monophosphate response elements binding protein (CREB) intracellular signaling pathway in the hippocampus. In total, 45 adult female Sprague-Dawley rats were randomly divided into three groups, namely the sham group, the middle cerebral artery occlusion (MCAO) group and the MCAO + EA group. Rats in the MCAO and MCAO + EA groups were modeled for post-stroke cognitive impairment. EA was performed at the Baihui and Shenting acupuncture points for $30 \mathrm{~min} /$ day for one week in the MCAO + EA group. Behavioral testing was analyzed using a step-down apparatus, while 2,3,5-triphenyl tetrazolium chloride was used to detect the infarct volume and lesion size. In addition, $\mathrm{CaM}$ activity was assessed by cyclic nucleotide-dependent phosphodiesterase analysis, and the protein expression levels of CaM, CaMKIV, phosphorylated (p)-CaMKIV, CREB and p-CREB were analyzed by western blot analysis. The cerebral I/R injured rat model in
\end{abstract}

Correspondence to: Dr Li-Dian Chen, Fujian Key Laboratory of Exercise Rehabilitation, Fujian University of Traditional Chinese Medicine, 1 Huatuo Road, Fuzhou, Fujian 350122, P.R. China

E-mail: chelidian@yahoo.com

${ }^{*}$ Contributed equally

Key words: electroacupuncture, cognitive deficits, cerebral ischemia-reperfusion, calmodulin, calmodulin-dependent protein kinase type IV, cyclic adenosine monophosphate response elements binding protein, hippocampus the MCAO group was established successfully with regard to the infarct volume and neuronal lesion size, as compared with the sham group. EA was demonstrated to effectively improve the cognitive ability, as measured by the step-down apparatus test, and decrease the infarct volume when compared with the MCAO group $(\mathrm{P}<0.05)$. The step-down apparatus test for the EA-treated rats revealed improved learning and reduced memory impairment when compared with the MCAO group. Furthermore, CaM activity and $\mathrm{CaM}$ protein expression levels in the $\mathrm{MCAO}+\mathrm{EA}$ group were lower compared with those in the MCAO group $(\mathrm{P}<0.05)$. By contrast, the protein expression levels of CaMKIV, p-CaMKIV, CREB and p-CREB were significantly reduced in the MCAO group when compared with the sham group $(\mathrm{P}<0.05)$, although the expression levels increased following EA treatment when compared with the MCAO group $(\mathrm{P}<0.05)$. Therefore, cognitive repair benefited from EA, and the main intracellular signaling pathway in the hippocampus was mediated by CaM-CaMKIV-CREB. EA effectively inhibited the expression and activity of CaM, while further enhancing the expression of CaMKIV and CREB, and their associated phosphorylated functions.

\section{Introduction}

Electroacupuncture (EA) is a therapy for the treatment of neurological dysfunction that functions by stimulating specific areas in the body. This therapeutic method originated in China $>2,000$ years ago, and over time EA has been accepted as conventional medicine in clinical practice worldwide (1-4). Statistical analyses of clinical results have indicated that the effects of EA on strokes are significant (5). Stroke is a major cause of several complications, including cognitive impairment, with $\sim 25 \%$ of patients suffering from cognitive impairment three months after a stroke (6). In addition, up to $75 \%$ of stroke survivors may be considered to have selective cognitive impairment, which commonly involves memory, orientation, language and attention (7). 
Cognitive rehabilitation and medication have been used to enhance cognition in patients who have had a stroke $(8,9)$; however, there is no one reliable method or medication that has been demonstrated in clinical practice. An increasing number of clinical trials have revealed that acupuncture exhibits positive effects following stroke, not only as a complementary and alternative medicine for post-stroke rehabilitation, but also as a preventative strategy that may induce cerebral ischemic tolerance (10-12).

Although the detailed mechanism underlying cognitive impairment remains unclear, neuronal excitotoxicity, the over-release of toxic neural transmitters and neuronal apoptosis have been demonstrated to contribute to the pathological process $(8,9)$. Neuronal excitotoxicity is triggered by intrinsic or extrinsic stimuli, which eventually result in the activation of caspases and nucleases, subsequently causing cell destruction (13).

Excitotoxicity via calcium-permeable glutamate receptors is considered to be a critical trigger in ischemia-induced brain damage. Experimental models have revealed that excitotoxicity may be inhibited using glutamate receptor antagonists, such as the non-competitive N-methyl-D-aspartate type glutamate receptor (NMDAR) antagonist, MK-801 (14). In contrast to an NMDAR blockade, the inhibition of specific postsynaptic NMDAR signaling by preconditioning neuroprotection may induce neuroprotection against cerebral ischemia-reperfusion (I/R) injury. Preconditioning has been associated with increased phosphorylation of cyclic adenosine monophosphate (cAMP) response element-binding (CREB) protein (15) and CREB-dependent transcription (16). Phosphorylation of CREB at Ser133 is involved in the regulation of neuronal plasticity and memory formation, and is required for glutamate- and $\mathrm{Ca}^{2+}$-dependent neuronal survival during development (17). In neurons of the central nervous system, CREB phosphorylation is induced by the synaptic activation of NMDARs, which occurs downstream of $\mathrm{Ca}^{2+} / \mathrm{CaM}$-dependent protein kinase (CaMK) activation (18). CaMKII and CaMKIV are able to regulate CREB activity; however, CaMKIV is specifically associated with the activation and transcription of trophic CREB (19). CaMKIV is a nuclear serine/threonine kinase that phosphorylates CREB at Ser133 and its transcription partner, CREB binding protein (20), thereby activating trophic gene transcription. Therefore, these observations indicate that CaM, CaMKIV and CREB activation are important for neuronal survival.

A previous study demonstrated that EA directly affected the biochemical materials associated with neuronal survival, including $\mathrm{Ca}^{2+}$, glutamate and NMDA; however, the detailed mechanisms underlying the intracellular signaling pathway in the hippocampus are yet to be fully elucidated (21). In the present study, EA was hypothesized to improve cognitive impairment in cerebral I/R-injured rats by adjusting the CaM-CaMKIV-CREB intracellular signaling function in the hippocampus.

\section{Materials and methods}

Animals. In total, 45 female Sprague Dawley rats (weight, $270 \pm 20 \mathrm{~g}$ ) were obtained from Shanghai SLAC Laboratory Animal Co. Ltd. (Shanghai, China) and housed in the Animal
Center of Fujian University of Traditional Chinese Medicine (TCM; Fuzhou, China). All the animals were housed under pathogen-free conditions with a 12-h light/dark cycle and free access to food and water. The experimental protocol was approved by the Institutional Animal Care and Use Committee of Fujian University of TCM.

The 45 rats were randomly divided into three groups, which included the sham group (rats underwent sham surgery), the middle cerebral artery occlusion (MCAO) group (rats underwent $\mathrm{MCAO}$ ) and the $\mathrm{MCAO}+\mathrm{EA}$ group (rats underwent $\mathrm{MCAO}$ and received EA intervention).

Establishment of the cerebral I/R injured rat model. Following overnight fasting, the rats were anesthetized with $10 \%$ chloral hydrate (3 ml/kg; Sigma-Aldrich, St. Louis, MO, USA) through intraperitoneal injection. Subsequently, $0.1000 .149 \mathrm{~mm}$ nylon surgical thread (Wego Holding Co., Ltd., Weihai, China) was inserted into the left internal carotid artery to block the middle cerebral artery when the blunted distal end met resistance. Following $2 \mathrm{~h}$ of occlusion, the thread was removed to allow complete reperfusion of the ischemic area. A sham procedure was carried out as aforementioned, without the occlusion of the middle cerebral artery $(21,22)$.

EA intervention. At $2 \mathrm{~h}$ after the surgery, rats in the $\mathrm{MCAO}+\mathrm{EA}$ group received EA treatment for $30 \mathrm{~min}$. The complete treatment period was 7 days. Baihui and Shenting acupuncture points in the governing vessel were selected for this study. Needles ( $0.3 \mathrm{~mm}$ diameter) were inserted to a depth of 2-3 mm and connected with the EA device (Model G6805; SMIF, Shanghai, China) with a disperse wave of 1 and $20 \mathrm{~Hz}$.

Step-down avoidance test. In the step-down inhibitory avoidance task, a rat is placed on an elevated platform in a dark compartment $(20 \times 20 \times 60 \mathrm{~cm})$, adjacent to a wall of an arena. When the rat steps down and places four paws onto the arena-floor grid, the rat receives a mild foot shock (36 V) and learns to associate the exploration of the arena with the punishment. On subsequent exposure to the same environment, the animal may increase the latency prior to 'stepping down' onto the floor grid, or may avoid stepping down. Rats were habituated to the handling procedure on the day prior to the test for $3 \mathrm{~min}$. Following any intervention, the rats were placed onto the platform again. The first time-period spent prior to stepping down onto the grid (latency period) and the frequency (number of errors) of stepping down from the platform within 3 min were recorded. In cases where the rats did not step-down from the platform within $3 \mathrm{~min}$, the number of errors was recorded as ' 0 ', and the latency period was '180 sec'. Step-down latencies and errors were recorded as a measure of memory retention (23-25).

Histopathological staining with 2,3,5-triphenyl tetrazolium chloride (TTC). Rats from each group were decapitated following anesthetization with $10 \%$ chloral hydrate $(3 \mathrm{ml} / \mathrm{kg})$. The brains were immediately removed and placed in ice/water at $-20^{\circ} \mathrm{C}$ for $15 \mathrm{~min}$ to ensure rigidity. Subsequently, the brains were cut into coronal sections of 2-mm thickness at the middle of the connecting line between the prefrontal cortex and the optic chiasma, after which the samples were immersed in 
A

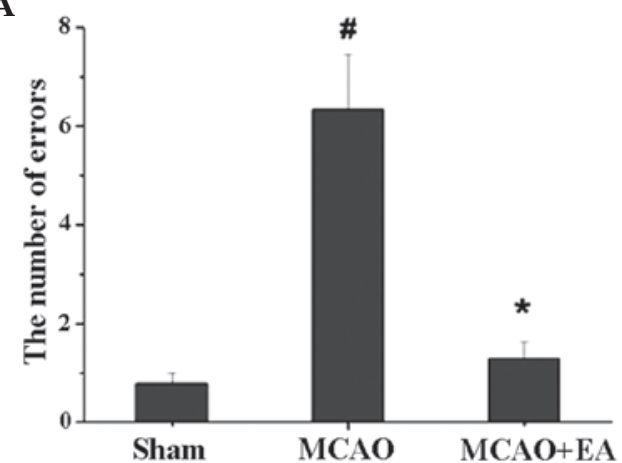

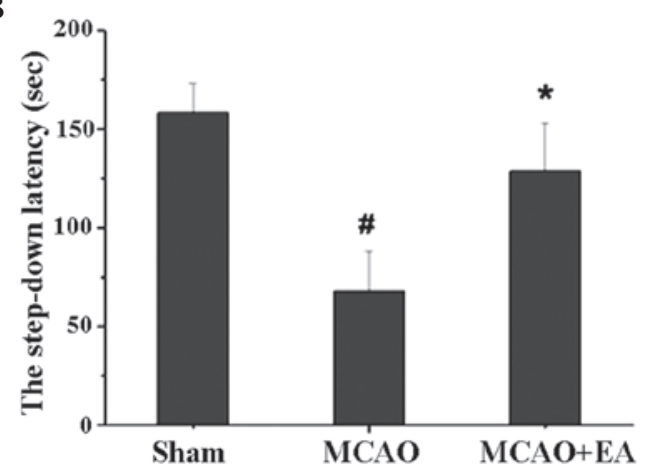

Figure 1. Results from the step-down avoidance test in each group. (A) Number of errors by the rats during the 3-min test. (B) Step-down latency of the rats. ${ }^{\text {"}} \mathrm{P}<0.05$, vs. sham group; ${ }^{\mathrm{P}}<0.05$, vs. MCAO group. MCAO, middle cerebral artery occlusion; EA, electroacupuncture.

$2 \%$ TTC (Sigma-Aldrich) (to avoid light) for $15 \mathrm{~min}\left(37^{\circ} \mathrm{C}\right)$ and treated with $4 \%$ paraformaldehyde (Sigma-Aldrich) for fixation for $24 \mathrm{~h}$. Finally, images of the brains were captured by camera (SX20; Canon, Inc., Tokyo, Japan). Analysis of the ischemia cerebral damage was performed as described previously $(22,26)$.

Phosphodiesterase (PDE) activity. A modified three-step PDE-1 assay was used to determine the CaM-dependent activation of PDE-1. In the PDE-1 assay, 3,5-cyclic-nucleotide PDE, 2-mM cAMP and $100 \mu \mathrm{M}$ CaM (Sigma-Aldrich) in $0.5 \mathrm{ml}$ Tris buffer solution (40 mM Tris-chloride, $0.1 \mathrm{mM}$ $\mathrm{MnCl}_{2}$ and $0.01 \mathrm{mM} \mathrm{CaCl}_{2}$ in distilled water at $\mathrm{pH} 7.5$ ) were incubated with increasing concentrations of native tehranolide $\left(1 \times 10^{-6}-9 \times 10^{-6} \mathrm{M}\right)$ for $10 \mathrm{~min}$ at $30^{\circ} \mathrm{C}$. The reaction was stopped by placing the test tubes in a boiling water bath for $2 \mathrm{~min}$, and then cooling. The 5-AMP in the reaction product was cleaved into adenosine and inorganic phosphate by incubation with 5'-nucleotidase $(100 \mu \mathrm{l}$; Sigma-Aldrich) for $10 \mathrm{~min}$, and the reaction was terminated by adding $0.05 \mathrm{ml}$ trichloroacetic acid $(55 \% \mathrm{w} / \mathrm{v})$ and $0.15 \mathrm{ml}$ molybdic acid solution, and centrifuging (10,800 x g) until clear. The clear supernatants were decanted into test tubes with Fiske-Subbarow reagent (Sigma-Aldrich). A blue color reaction was allowed to develop in the presence of inorganic phosphorus for $10 \mathrm{~min}$, and the absorbance was measured at $660 \mathrm{~nm}$ using spectrophotometry (SmartSpec Plus; Bio-Rad Laboratories, Inc., Hercules, CA, USA) (27-29).

Western blot analysis for the determination of CaM, CaMKIV, phosphorylated (p)-CaMKIV, CREB and p-CREB protein expression levels. Hippocampi from three groups were homogenized in nondenaturing lysis buffer [20 mM Tris ( $\mathrm{pH} 7.5)$, $150 \mathrm{mM} \mathrm{NaCl}, 1 \%$ Triton X-100, $1 \%$ NP-40, 2 mM sodium pyrophosphate, $25 \mathrm{mM} \beta$-glycerophosphate, $1 \mathrm{mM}$ EDTA, $1 \mathrm{mM}$ $\mathrm{Na}_{3} \mathrm{VO}_{4}, 0.5 \mu \mathrm{g} / \mathrm{ml}$ leupeptin) and centrifuged at $12000 \mathrm{x} \mathrm{g}$ for $15 \mathrm{~min}$. The supernatants were collected and frozen at $-80^{\circ} \mathrm{C}$ prior to immunoblotting. Protein concentration was determined using a Bio-Image Analysis System (ChemiDoc ${ }^{\mathrm{TM}}$ Imaging Systems; Bio-Rad Laboratories, Inc.). In total, a 50- $\mu$ g protein sample obtained from the CA1 region of the hippocampus was loaded onto a $12 \%$ SDS-PAGE gel. Following electrophoresis, the proteins were electrotransferred onto polyvinylidene difluoride membranes (Sigma-Aldrich). The blots were blocked with $5 \%$ non-fat milk for $2 \mathrm{~h}$, and subsequently incubated with primary antibodies (1:1,000 dilution) against $\mathrm{CaM}$ (cat. no. sc-137079; Santa Cruz Biotechnology, Inc., Dallas, TX, USA), CaMKIV (cat. no. 4032; Cell Signaling Technology, Inc., Danvers, MA, USA), p-CaMKIV (cat. no. sc-28443-R; Santa Cruz Biotechnology, Inc), CREB (cat. no. 9197; Cell Signaling Technology, Inc.), p-CREB (cat. no. 9196; Cell Signaling Technology, Inc.) and $\beta$-actin (cat. no. 4970; Cell Signaling Technology, Inc.) overnight at $4^{\circ} \mathrm{C}$. Next, the blots were incubated with a horseradish peroxidase-conjugated anti-rabbit or anti-mouse secondary antibodies (1:5,000; cat. nos. 7074 and 7076; Cell Signaling Technology, Inc.) for $50 \mathrm{~min}$. $\beta$-actin was used as a loading control. The blots were developed using a commercially available enhanced chemiluminescence kit (Bio-Rad Laboratories, Inc.), and examined using a Bio-Image Analysis System (ChemiDoc ${ }^{\mathrm{TM}}$ Imaging systems; Bio-Rad Laboratories, Inc.) (30).

Statistical analysis. Data are presented as the mean \pm standard error of the mean. Statistical comparisons were conducted by one way analysis of variance using the SPSS software package, version 18.0 (SPSS, Inc., Chicago, IL, USA), where $\mathrm{P}<0.05$ was considered to indicate a statistically significant difference.

\section{Results}

Effect of EA on the step-down avoidance test and infarct volume. As shown in Fig. 1, MCAO markedly affected the memory of the rats, while EA treatment was shown to successfully repair this ability. The step-down latency of the MCAO group rats was significantly shorter when compared with the sham group $(\mathrm{P}<0.05)$, and was prolonged by EA $(\mathrm{P}<0.05)$. During the 3-min test, the number of errors in the MCAO group rats was significantly higher when compared with the sham group and MCAO + EA group rats (Fig. 1; $\mathrm{P}<0.05$ ).

With regard to the infarct volume analyses, EA was revealed to significantly reduce the infarct volume caused by cerebral I/R. The sham group rats exhibited no trauma in the brain, while the MCAO group rats exhibited a large infarct area $(23.98 \pm 5.04 \%$; $\mathrm{P}<0.05)$, which was significantly decreased following $\mathrm{EA}$ (15.71 $\pm 3.16 \%$; $\mathrm{P}<0.05$; Fig. 2).

Effect of EA on the levels of CaM activity and CaM protein expression. Notably, MCAO was found to promote CaM activity 
A

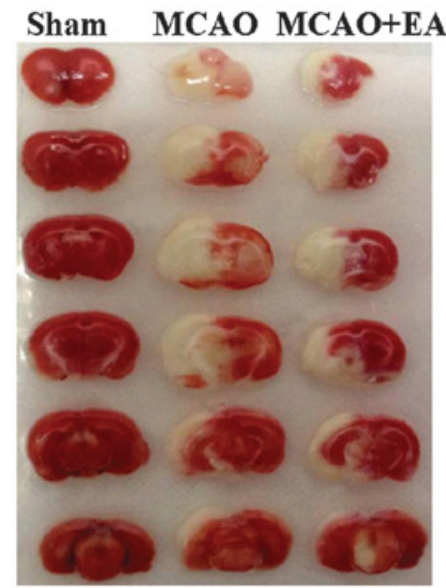

B

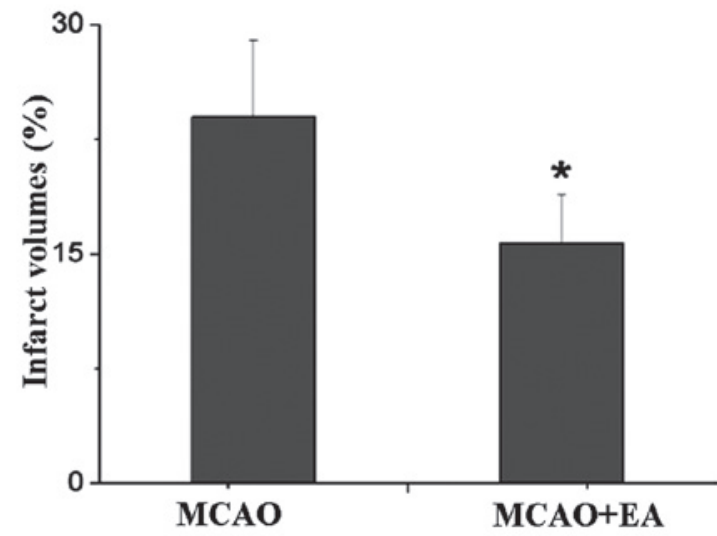

Figure 2. Histopathological examinations in each group. (A) Pathological features of cerebral ischemia, where red tissues indicate normal tissue and white sections indicate infarction. (B) Quantitative analysis of the infarct volume comparison between the MCAO and MCAO + EA groups. "P<0.05, vs. MCAO group. MCAO, middle cerebral artery occlusion; EA, electroacupuncture.

A

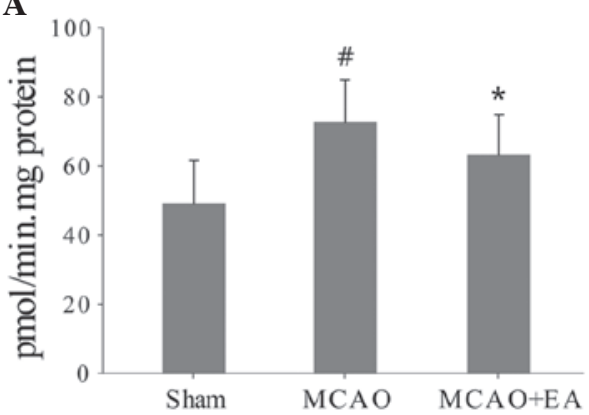

B

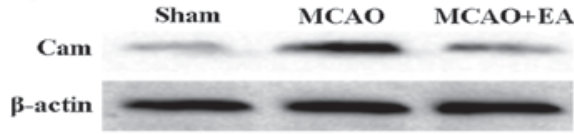

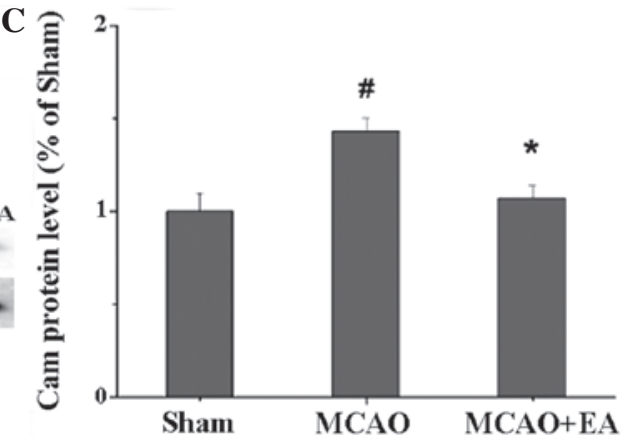

Figure 3. (A) Activity levels and (B) protein expression levels of CaM in each group. (C) Quantitative analyses of the protein expression levels of CaM, which were in accordance with the western blot analysis results. ${ }^{\text {}} \mathrm{P}<0.05$, vs. sham group; ${ }^{*} \mathrm{P}<0.05$, vs. MCAO group. CaM, calmodulin; MCAO, middle cerebral artery occlusion; EA, electroacupuncture.

A

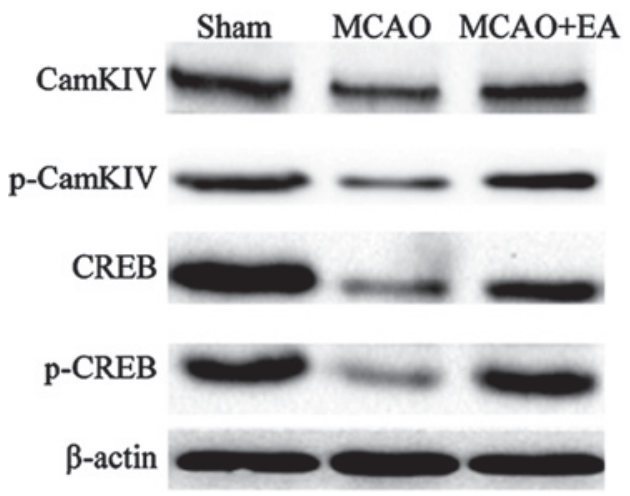

B

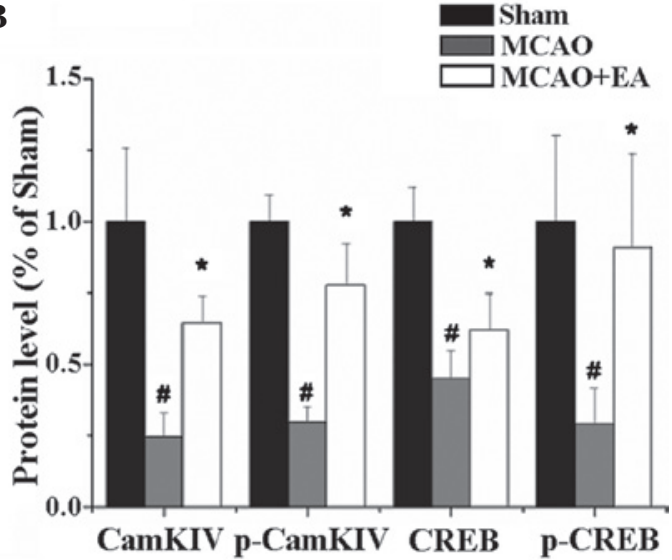

Figure 4. (A) Protein expression levels of CaMKIV, p-CaMKIV, CREB and p-CREB in each group. (B) Quantitative analyses of the protein expression levels of CaMKIV, p-CaMKIV, CREB and p-CREB, which were in accordance with the western blot analysis results. ${ }^{\prime \prime} \mathrm{P}<0.05$, vs. sham group; ${ }^{*} \mathrm{P}<0.05$, vs. MCAO group. CaM, calmodulin; MCAO, middle cerebral artery occlusion; EA, electroacupuncture; CaMKIV, calmodulin-dependent protein kinase type IV; CREB, cyclic adenosine monophosphate response elements binding protein; p-CREB, phosphorylated CREB; p-CaMKIV, phosphorylated CaMKIV.

and protein expression, whereas EA reduced these reactions. According to the PDE analyses, CaM activity was promoted by MCAO and inhibited by EA $(\mathrm{P}<0.05)$, and the same result was observed for $\mathrm{CaM}$ protein expression $(\mathrm{P}<0.05 ;$ Fig. 3 ).
Effect of EA on the protein expression levels of CaMKIV, $p$-CaMKIV, CREB and p-CREB. Protein expression levels of CaMKIV, p-CaMKIV, CREB and p-CREB were shown to decrease following MCAO and increase with EA treatment. 
MCAO severely inhibited the protein expression of CaMKIV and pCaMKIV in the hippocampus $(\mathrm{P}<0.05)$, while EA repaired the expression of these proteins and promoted CaMKIV and CREB phosphorylation $(\mathrm{P}<0.05)$. Furthermore, CREB and $\mathrm{p}-\mathrm{CREB}$ presented a similar variation trend where MCAO reduced CREB and $\mathrm{p}-\mathrm{CREB}$ expression $(\mathrm{P}<0.05)$, while EA promoted their expression $(\mathrm{P}<0.05$; Fig. 4).

\section{Discussion}

Therapeutics in clinical stroke treatment has led researchers to question the feasibility of neuroprotection. Novel insights into the cellular events responsible for neuronal death and an improved understanding of the toxic and trophic roles of excitatory neurotransmission are creating new avenues for therapeutic research. The presence of protective signaling cascades downstream of NMDAR activation, such as enhanced antioxidant defenses, results in the suppression of proapoptotic signaling and the maintenance of trophic signal events (31).

In the present study, according to the pathological features of cerebral ischemia, where red tissues indicate normal tissue and white sections indicate infarction, EA was demonstrated to reduce the infarct volume. As the infarct volume decreased, the behavior study was estimated by the step-down avoidance test. Using this test in previous studies $(23,24,32)$, EA was shown to improve the memories of rats following stroke. Previous clinical studies and meta-analyses have demonstrated that EA exerts a positive effect on cognitive function when compared with no acupuncture, medicine or rehabilitation $(11,12,33)$. Furthermore, a series of clinical trials have shown that acupuncture regulates the release of neurochemicals, hemorheology, cerebral microcirculation, metabolism, neuronal activity, and the function of specific brain regions (10,34,35). Animal studies have revealed that the effects of acupuncture therapy on stroke may possibly be mediated through the inhibition of post-ischemic inflammatory reactions, the stimulation of neurogenesis and angiogenesis, and the influence on neural plasticity $(36,37)$.

Recently, improvement in cognitive dysfunction by EA following stroke has attracted increasing interest. Physical rehabilitation is not the only treatment aim, but also cognitive improvement is closely associated with the quality of daily life (38).

Transport of $\mathrm{Ca}^{2+} / \mathrm{CaM}$ from the surface membrane to the nucleus activates CaMK kinase (CaMKK) and the substrate, CaMKIV, the CREB kinase. This classical cellular signaling pathway is considered to be closely associated with cognitive function in the hippocampus. $\mathrm{Ca}^{2+}$ binding to $\mathrm{CaM}$, and the consequent activation of $\mathrm{Ca}^{2+} / \mathrm{CaM}$-dependent protein kinases combined with the CaM kinase family, contribute strongly to synaptic potentiation, learning and memory. Additional CaM kinases and CaMKIV form a CaMK cascade within the nucleus. Neuronal activity and $\mathrm{Ca}^{2+} / \mathrm{CaM}$ drive $\mathrm{CaMKK}$ to phosphorylate and activate nuclear CaMKIV, which phosphorylates CREB and CREB-binding protein (39). In the present study, a notable finding was that the expression and activity of $\mathrm{CaM}$ in the MCAO group was significantly increased, in contrast to the sham and EA groups. Thus, the pathological process is yet to be fully elucidated. However, the expression levels of the additional proteins varied as predicted. EA promoted CaMKIV, p-CaMKIV, CREB and p-CREB protein expression. According to a previous study, the inhibitory effect of EA on NF- $\kappa$ B activation led to the inhibition of cerebral cell apoptosis and an improved cognitive ability (21). Therefore, the CaM-CaMKIV-CREB pathway may be an additional important cellular signaling pathway involved in cognitive improvement.

The present study is preliminary investigation that used rat models; thus, the effect of EA on post-stroke cognitive impairment requires detailed evaluation in clinical practice. The aim of the present study was to explain the detailed mechanism underlying the effects of EA on cognitive impairment from a novel perspective; however, the mechanisms of EA on stroke are complex, comprehensive and wide. Therefore, this cellular signaling pathway may not be the only neuronal signaling pathway involved in cognitive impairment.

In conclusion, to the best of our knowledge, the present study is the first to demonstrate that EA exhibits excellent cognitive repair properties, with the underlying mechanism closely associated with the CaM-CaMKIV-CREB signaling pathway.

\section{Acknowledgements}

This study was supported by a grant from the National Natural Science Foundation of China (no. 81273835).

\section{References}

1. Sator-Katzenschlager SM and Michalek-Sauberer A: P-Stim auricular electroacupuncture stimulation device for pain relief. Expert Rev Med Devices 4: 23-32, 2007.

2. Toosizadeh N, Lei H, Schwenk M, Sherman SJ, Sternberg E, Mohler J and Najafi B: Does integrative medicine enhance balance in aging adults? Proof of concept for the benefit of electroacupuncture therapy in Parkinson's disease. Gerontology 61: 3-14, 2015.

3. Yu HJ, Zhu JG, Shen P, Shi LH, Shi YC and Chen F: Electroacupuncture decreases the urinary bladder pressure in patients with acute gastrointestinal injury. Genet Mol Res 14: 34-39, 2015.

4. Zyloney CE, Jensen K, Polich G, Loiotile RE, Cheetham A, LaViolette PS, Tu P, Kaptchuk TJ, Gollub RL and Kong J: Imaging the functional connectivity of the Periaqueductal Gray during genuine and sham electroacupuncture treatment. Mol Pain 6: 80, 2010.

5. Inoue I, Fukunaga M, Koga K, Wang HD and Ishikawa M: Scalp acupuncture effects of stroke studied with magnetic resonance imaging: Different actions in the two stroke model rats. Acupunct Med 27: 155-162, 2009

6. Haring HP: Cognitive impairment after stroke. Curr Opin Neurol 15: 79-84, 2002.

7. Cumming TB, Marshall RS and Lazar RM: Stroke, cognitive deficits, and rehabilitation: Still an incomplete picture. Int J Stroke 8: $38-45,2013$

8. Hachinski V and Munoz D: Vascular factors in cognitive impairment - where are we now? Ann N Y Acad Sci 903: 1-5, 2000.

9. Tatemichi TK, Desmond DW, Stern Y, Paik M, Sano M and Bagiella E: Cognitive impairment after stroke: Frequency, patterns and relationship to functional abilities. J Neurol Neurosurg Psychiatry 57: 202-207, 1994.

10. Li X and Wang Q: Acupuncture therapy for stroke patients. Int Rev Neurobiol 111: 159-179, 2013.

11. Li L, Zhang H, Meng SQ and Qian HZ: An updated meta-analysis of the efficacy and safety of acupuncture treatment of cerebral infarction. PLoS One 9: el14057, 2014.

12. Shih CC, Hsu YT, Wang HH, Chen TL, Tsai CC, Lane HL, Yeh CC, Sung FC, Chiu WT, Cherng YG and Liao CC: Decreased risk of stroke in patients with traumatic brain injury receiving acupuncture treatment: A population-based retrospective cohort study. PLoS One 9: e89208, 2014. 
13. Cory S and Adams JM: The Bcl2 family: Regulators of the cellular life-or-death switch. Nat Rev Cancer 2: 647-656, 2002.

14. Davis SM, Lees KR, Albers GW, Diener HC, Markabi S, Karlsson G and Norris J: Selfotel in acute ischemic stroke: Possible neurotoxic effects of an NMDA antagonist. Stroke 31: 347-354, 2000

15. Meller R, Minami M, Cameron JA, Impey S, Chen D, Lan JQ, Henshall DC and Simon RP: CREB-mediated Bcl-2 protein expression after ischemic preconditioning. J Cereb Blood Flow Metab 25: 234-246, 2005.

16. Kitagawa K: CREB and cAMP response element-mediated gene expression in the ischemic brain. FEBS J 274: 3210-3217, 2007.

17. Ao H, Ko SW and Zhuo M: CREB activity maintains the survival of cingulate cortical pyramidal neurons in the adult mouse brain. Mol Pain 2: 15, 2006.

18. Deisseroth K, Bito H and Tsien RW: Signaling from synapse to nucleus: Postsynaptic CREB phosphorylation during multiple forms of hippocampal synaptic plasticity. Neuron 16: 89-101, 1996

19. Bok J, Wang Q, Huang J and Green SH: CaMKII and CaMKIV mediate distinct prosurvival signaling pathways in response to depolarization in neurons. Mol Cell Neurosci 36: 13-26, 2007.

20. Impey S, Fong AL, Wang Y, Cardinaux JR, Fass DM, Obrietan K, Wayman GA, Storm DR, Soderling TR and Goodman RH: Phosphorylation of CBP mediates transcriptional activation by neural activity and CaM kinase IV Neuron 34: 235-244, 2002.

21. Feng X, Yang S, Liu J, Huang J, Peng J, Lin J, Tao J and Chen L: Electroacupuncture ameliorates cognitive impairment through inhibition of NF- $\mathrm{BB}$-mediated neuronal cell apoptosis in cerebral ischemia-reperfusion injured rats. Mol Med Rep 7: 1516-1522, 2013

22. Xue X, You Y, Tao J, Ye X, Huang J, Yang S, Lin Z, Hong Z, Peng $J$ and Chen L: Electro-acupuncture at points of Zusanli and Quchi exerts anti-apoptotic effect through the modulation of PI3K/Akt signaling pathway. Neurosci Lett 558: 14-19, 2014.

23. Zhu L, Zhang L, Zhan LB, Lu X, Peng J, Liang L, Liu Y, Zheng L, Zhang F and Liu Q: The effects of Zibu Piyin Recipe components on scopolamine-induced learning and memory impairment in the mouse. J Ethnopharmacol 151: 576-582, 2014.

24. Tsapakis EM, Fernandes C, Moran-Gates T, Basu A, Sugden K, Aitchison KJ and Tarazi FI: Effects of antidepressant drug exposure on gene expression in the developing cerebral cortex. Synapse 68: 209-220, 2014

25. Heo YM, Shin MS, Lee JM, Kim CJ, Baek SB, Kim KH and Baek SS: Treadmill exercise ameliorates short-term memory disturbance in scopolamine-induced amnesia rats. Int Neurourol J 18: 16-22, 2014.
26. Zhou H, Zhang Z, Wei H, Wang F, Guo F, Gao Z, Marsicano G, Wang Q and Xiong L: Activation of STAT3 is involved in neuroprotection by electroacupuncture pretreatment via cannabinoid CB1 receptors in rats. Brain Res 1529: 154-164, 2013.

27. Tan H, West JA, Ramsay JP, Monson RE, Griffin JL, Toth IK and Salmond GP: Comprehensive overexpression analysis of cyclic-di-GMP signalling proteins in the phytopathogen Pectobacterium atrosepticum reveals diverse effects on motility and virulence phenotypes. Microbiology 160: 1427-1439, 2014.

28. Liu CP, Kuo MS, Wu BN, Chai CY, Huang HT, Chung PW and Chen IJ: NO-releasing xanthine KMUP-1 bonded by simvastatin attenuates bleomycin-induced lung inflammation and delayed fibrosis. Pulm Pharacol Ther 27: 17-28, 2014.

29. Bowman PB and Puett D: Electron paramagnetic resonance spectroscopy of nitroxide-labeled calmodulin. Protein J 33: 267-277, 2014

30. Willard SS and Koochekpour S: Glutamate, glutamate receptors and downstream signaling pathways. Int J Biol Sci 9: 948-959, 2013.

31. Balazs R: Trophic effect of glutamate. Curr Top Med Chem 6: 961-968, 2006.

32. Jing XH, Chen SL, Shi H, Cai H and Jin ZG: Electroacupuncture restores learning and memory impairment induced by both diabetes mellitus and cerebral ischemia in rats. Neurosci Lett 443: 193-198, 2008

33. Liu F, Li ZM, Jiang YJ and Chen LD: A meta-analysis of acupuncture use in the treatment of cognitive impairment after stroke. J Altern Complem Med 20: 535-544, 2014.

34. Chu Q, Wang L and Liu GZ: Effect of acupuncture on hemorheology in patients with diabetic nephropathy. Zhen $\mathrm{Ci}$ Yan Jiu 32: 335-337, 2007 (In Chinese).

35. Zhang SQ, Wang YJ, Zhang JP, Chen JQ, Wu CX, Li ZP, Chen JR, Ouyang HL, Huang Y and Tang CZ: Brain activation and inhibition after acupuncture at Taichong and Taixi: Resting-state functional magnetic resonance imaging. Neural Regen Res 10: 292-297, 2015.

36. Zhang C, Wen Y, Fan X, Yang S, Tian G, Zhou X, Chen Y and Meng Z: A microarray study of middle cerebral occlusion rat brain with acupuncture intervention. Evid Based Complement Alternat Med 2015: 496932, 2015.

37. Leung MC, Yip KK, Lam CT, Lam KS, Lau W, Yu WL, Leung AK and So KF: Acupuncture improves cognitive function: A systematic review. Neural Regen Res 8: 1673-1684, 2013.

38. Chou PC, Chu HY and Lin JG: Effects of electroacupuncture treatment on impaired cognition and quality of life in Taiwanese stroke patients. J Altern Complem Med 15: 1067-1073, 2009.

39. Ma H, Groth RD, Cohen SM, Emery JF, Li B, Hoedt E, Zhang G, Neubert TA and Tsien RW: $\gamma \mathrm{CaMKII}$ shuttles $\mathrm{Ca}(2+) / \mathrm{CaM}$ to the nucleus to trigger CREB phosphorylation and gene expression. Cell 159: 281-294, 2014. 